\title{
Recommend-Response and Complications Comparing Radiofrequency Ablation Versus Parathyroidectomy for Secondary Hyperparathyroidism in Dialysis Patients
}

\author{
Mian Ren \\ Hangzhou Medical College \\ Danna Zheng \\ Hangzhou Medical College \\ Juan Wu \\ Hangzhou Medical College \\ Yueming Liu \\ Hangzhou Medical College \\ Chengzhong Peng \\ Hangzhou Medical College \\ Wei Shen \\ Hangzhou Medical College \\ Bo Lin ( $\nabla$ doctor_linbo@163.com ) \\ Hangzhou Medical College
}

\section{Research Article}

Keywords: complications, radiofrequency, parathyroidectomy, hyperparathyroidism

Posted Date: December 28th, 2021

DOI: https://doi.org/10.21203/rs.3.rs-1145358/v1

License: (c) (1) This work is licensed under a Creative Commons Attribution 4.0 International License. Read Full License 


\section{Abstract \\ Introduction}

: To compare the efficacy and safety of ultrasound(US)-guided radiofrequency ablation (RFA) and parathyroidectomy (PTX) in the treatment of secondary hyperparathyroidism (SHPT).

\section{Methods}

In this retrospective study, we divided patients into PTX $(n=53)$ and RFA $(n=47)$ groups. The primary outcomes were the proportion of patients achieved the target range of iPTH concentrations(124$558 \mathrm{pg} / \mathrm{mL}$ ) and the long-term prognosis. The secondary outcomes were the differences in the changes of iPTH, calcium, and phosphorus over time and the incidence of adverse events.

\section{Results}

There was rarely difference in baseline characteristics between the two groups. Primary outcomes: The iPTH concentrations of $25.8 \%$ of patients in the PTX group and $51.3 \%$ of the RFA group were within the recommended range at the endpoint $(P=0.031)$. Survival analysis revealed that the difference in all-cause mortality and cumulative response rate between the two groups was not statistically significant $(P=0.902$, $\mathrm{P}=0.141$, respectively). Secondary outcomes: The iPTH concentrations in PTX group and RFA group dropped sharply after treatment and were $82.30 \pm 163.21 \mathrm{pg} / \mathrm{mL}$ and $279.96 \pm 306.57 \mathrm{pg} / \mathrm{mL}(P<0.001)$. There was no difference in the trend of $\mathrm{iPTH}$, calcium, and phosphorus levels between the two groups over time $(P>0.05)$. In addition, the incidence of infection and the hospital stay in the RFA group were significantly less. And preoperative bone-specific alkaline phosphatase concentration was a risk factor for postoperative hypocalcemia.

\section{Conclusion}

US-guided RFA was minimally invasive and no less than PTX in terms of cumulative response rate and complications in the treatment of severe SHPT in maintenance dialysis patients and may be used as an alternative technique to PTX, which need further studies to confirm.

\section{Introduction}

Persistent calcium-phosphorus-vitamin D metabolism disorders and increased levels of fibroblast growth factor 23(FGF-23) in chronic kidney disease(CKD) stimulate the excessive secretion of parathyroid hormone(PTH), triggering secondary hyperplasia or adenoma formation in parathyroid gland tissue, namely secondary hyperparathyroidism(SHPT $)^{1}$. SHPT contributes to bone pain and fracture, accelerates 
vascular calcification, and decreases life quality, is one of the main risk factors for death and cardiovascular events in patients with end-stage renal disease(ESRD) ${ }^{2}$.

At present, the drugs used to treat hyperparathyroidism are mainly active vitamin $D$ and calcimimetics ${ }^{3,4}$. There are certain limitations due to adverse reactions or drug resistance, and active vitamin $D$ is ineffective for already formed parathyroid adenomas. Therefore, the standard therapy for severe druginvalid SHPT is parathyroidectomy(PTX $)^{1,3,4}$. Total parathyroidectomy may lead to permanent hypoparathyroidism and adynamic bone disease, requiring long-term calcium and calcitriol supplementation ${ }^{5}$.

However, for patients with multiple complications who cannot tolerate PTX, ultrasound intervention such as microwave ablation(MWA) ${ }^{6,7}$ and radiofrequency ablation(RFA $)^{8,9}$ of parathyroid glands can be selected. Ultrasound-guided percutaneous ablation therapy is minimally invasive and reproducible, and has been more widely used to treat hyperparathyroidism. Some previous studies showed that MWA or RFA could indeed reduce PTH levels and is effective for SHPT ${ }^{10-12}$, and are not inferior to PTX in safety and efficacy $7,12-15$, but the comparisons of PTX and RFA in hospital stay, economic costs, and especially long-term results are less.

In addition, due to the sharp drop in PTH after operation, the rapid intake of calcium by bones leads to "hungry bone syndrome" ${ }^{16}$. Severe hypocalcemia(SH) ${ }^{17}$ will cause convulsion, myocardial dysfunction, seizures, and even sudden death. Therefore, maintaining normal postoperative blood calcium is very important. Few studies have shown that preoperative alkaline phosphatase(ALP) ${ }^{18}$ and $\mathrm{PTH}^{19}$ levels are independent risk factors for postoperative SH in SHPT patients ${ }^{5,20}$.

The aim of our retrospective study was to compare the clinical outcomes, postoperative complications and long-term prognosis of PTX and RFA in maintenance dialysis patients with SHPT.

\section{Materials And Methods}

Patients.

This study was approved by the ethical and scientific review board of Zhejiang Provincial People's Hospital. We confirm that all methods were performed in accordance with the relevant guidelines and regulations of declarations of Helsinki. The retrospective cohort study included all patients diagnosed as SHPT who underwent PTX or ultrasound-guided RFA from June 2014 to December 2020 in our hospital. The inclusion criteria were (1) 18-85 years old; (2) dialytic vintage $\geq 6$ months; (3) postoperative iPTH concentration $>600 \mathrm{pg} / \mathrm{mL}$; (4) severe SHPT after ineffective medical treatment; (5) at least one hyperplastic parathyroid nodules with diameter $\geq 1 \mathrm{~cm}$ found by ultrasound examination; (6) follow-up durations $\geq 3$ months; The exclusion criteria were: (1) primary or tertiary hyperparathyroidism; (2) severe cardiopulmonary insufficiency who can't tolerate treatment; (3) previous history of PTX or RFA; 
Intervention.

All patients underwent routine high-frequency ultrasound combined with 99mTc-sestamibi SPECT or enhanced CT evaluation for preoperative parathyroid localization. According to the clinical guidelines, combined with the patient's condition, the clinician judged and fully communicated 2 methods with the patients. Patients who cannot tolerate general anesthesia or preferred minimally invasive therapy accepted RFA. And they were assigned into 2 groups by the treatment: PTX group and RFA group.

Ultrasound(US)-guided RFA was performed by the same doctor who had rich experience in ultrasound intervention and RFA treatment of parathyroid nodules. After disinfection and local anesthesia, 10-30ml of sterile water was injected around parathyroid in order to establish a heat insulation layer to avoid thermal injury of adjacent tissues. Using iU22 US scanner and a high-frequency linear probe (L12-5) (Philips, The Netherlands) grey-scale imaging for guidance, while contrast-enhanced ultrasound (CEUS) with a high-frequency linear probe (L9-3) for monitoring, the needle of the 18G radiofrequency electrode (VIVA; STARmed, Goyang, Korea) with a 7mm active tip was inserted into the target parathyroid nodules to perform thermal ablation. During the procedure, the physician evaluated the patient's condition and terminated the operation immediately if hoarseness occurred. Postoperative management: Closely observe whether hematoma or asphyxia occurs. After 4 hours, patients gradually resumed eating. Routinely evaluate whether the patients have hoarseness and bucking. If the degree of iPTH decline is not satisfactory, further examination whether there is residual adenoma or ectopic parathyroid gland is needed.

Total PTX was performed after general anesthesia and routine disinfection. Patient was placed in a supine position. The anterior transverse neck incision was about $5 \mathrm{~cm}$ along the dermatoglyphic direction, the skin flaps were separated and fixed under the platysma, and the anterior cervical muscles were separated along the white line of the neck. After fully exposition of the bilateral thyroid and parathyroid glands, surgeon carefully dissected and protected the bilateral recurrent laryngeal nerves, then completely removed all visible parathyroid glands. Hemostasis was fully achieved and the anterior neck incision was sutured layer by layer. Parathyroid auto-transplantation(AT) was performed to cut about 30-60 mg of parathyroid tissue into $1 \mathrm{~mm} \times 1 \mathrm{~mm} \times 1 \mathrm{~mm}$ particles and planted on the forearm brachioradialis.

Clinical data collection.

General information of age, gender, dialysis history, clinical symptoms and treatment procedure were collected. Clinical laboratory variables at baseline such as serum creatinine(Cr), uric acid(UA), albumin(ALB), hemoglobin(Hb), troponin I(TNI), B-type natriuretic peptide(BNP), C-reactive protein(CRP), intact parathyroid hormone(iPTH), calcium(Ca), and phosphorus(P) levels, and bone metabolism related indicators such as bone-specific alkaline phosphatase(bALP), Beta C-terminal cross-linked telopeptides of type I collagen( $\beta$-CTx), N-terminal osteocalcin(N-MID), total type I collagen N-terminal propeptide(tP1NP), 25-hydroxy vitamin $\mathrm{D}(25(\mathrm{OH}) \mathrm{D})$, etc were collected. Clinical data including the number and size of parathyroid nodules, osteoporosis or not (bone density), carotid arteriosclerosis or not (carotid artery Bultrasound), hospital stay and postoperative complications. 
Follow-up and outcomes.

Serum iPTH, calcium, and phosphorus concentrations were conducted after PTX or RFA at the following time points: 1-month ( \pm 2 weeks), 3-month ( \pm 2 weeks), 6-month ( \pm 1 month), 12-month ( \pm 1 month), 24month ( \pm 1 month). All patients were followed up until death, kidney transplantation, loss or the end of the study (30 June 2021).

The primary outcomes were the proportion of patients in PTX group or RFA group with the target range of iPTH concentration during the efficacy assessment phase (follow-up to the endpoint), and the long-term prognosis (death or recurrence). According to the Kidney Disease Improving Global Outcomes(KDIGO) Guidelines $^{21}$, the target range of iPTH concentration was maintained at approximately 2 to 9 times the upper limit of normal, while goal achievement for iPTH of 124-558 pg/mL, calcium of 2.0-2.5 mmol/L, and phosphorus of 0.97-1.62 mmol/L. Cumulative response rate was defined as the proportion of patients with iPTH $<558 \mathrm{mmol} / \mathrm{L}$ for three consecutive months before endpoint of the study, while the preoperative clinical symptoms were completely relieved.

The secondary outcomes were the differences in the changes of iPTH, calcium, and phosphorus over time between the two groups, as well as the occurrence of postoperative adverse events (hoarseness, fever, hematoma, and hypocalcemia, etc.). Clinically, if serum calcium $<2.0 \mathrm{mmol} / \mathrm{L}$ was considered to be hypocalcemia, serum calcium $<1.8 \mathrm{mmol} / \mathrm{L}$ was $\mathrm{SH}$, initiating with intravenous calcium ${ }^{5,17}$. If serum calcium was between 1.8-2.1 mmol/L in the perioperative period, oral calcium carbonate $(1.8-5.4 \mathrm{~g} / \mathrm{d})$ and calcitriol $(1.0-2.5 \mu \mathrm{g} / \mathrm{d})$ were given.

\section{Statistical analysis.}

All statistical analyses were performed using SPSS version 26.0 for Mac and Graphpad Prism version 9.0. The measured data conforming to a normal distribution were displayed as the mean \pm standard deviation(SD), and the other data were displayed as the median and interquartile range. Comparisons between parameters were performed using the independent sample T-test, Mann-Whitney U test or Chisquared test. Potential predictors of hypocalcemia occurrence were analyzed by logistical regression analysis. Survival analyses were calculated by the Kaplan-Meier survival curve. All results were tested by bilateral tests, and significance was indicated by $\mathrm{P}<0.05$.

\section{Results}

\section{General information}

A total of 100 patients received therapy from June 2014 to December 2020 . There were 53 patients in the PTX group, of which 47 (88.7\%) underwent total PTX with AT; 6 (11.3\%) underwent total PTX, and a total of 47 patients in the RFA group, of which 26 (55.0\%) performed single-session RFA; 21 (45\%) received two-session RFA. 
The baseline characteristics of the patients are summarized in Table 1. The average age of these patients was $50.61 \pm 12.41$ years old. The average dialytic vintage was $7.77 \pm 3.60$ years. The median follow-up time was 30.025 (22.325-38.725) months. And $81 \%$ patients were undergoing hemodialysis. There was no statistical difference between the two groups in several variables such as age, gender, dialysis history, follow-up time, renal function, UA, ALB, Hb, CRP, TNI, BNP, iPTH, calcium, phosphorus, bALP, etc. $(P>0.05)$.

More parathyroid hyperplasia nodules were detected in the PTX group. There were $3.92 \pm 0.43$ nodules resected in the PTX group, $3.68 \pm 0.63$ nodules ablated in the RFA group $(P=0.027)$. But there was no difference in the nodule's maximum diameter and preoperative clinical symptoms (ostealgia or arthralgia, cutaneous pruritus, skeleton distortion, calcinosis cutis) between the two groups. The results of bonederived turnover markers, osteoporosis, and vascular calcification suggested the existence of chronic kidney disease related mineral and bone disorder(CKD-MBD) in both groups.

Table 1. Patients' baseline characteristics. 


\begin{tabular}{|c|c|c|c|}
\hline Parameter & PTX Group $(n=53)$ & RFA Group $(n=47)$ & $P$ value \\
\hline Age(years) & $50.36 \pm 12.73$ & $50.89 \pm 12.17$ & 0.831 \\
\hline Genger, male & $66.0 \%$ & $61.7 \%$ & 0.652 \\
\hline Dialysis method, Hemodialysis & $83.0 \%$ & $78.7 \%$ & 0.585 \\
\hline Dialytic vintage (years) & $7.85 \pm 3.76$ & $7.69 \pm 3.45$ & 0.824 \\
\hline Follow-up time (months) & $31.70(23.40-42.70)$ & $28.60(21.30-36.60)$ & 0.085 \\
\hline Nodule numbers & $3.92 \pm 0.43$ & $3.68 \pm 0.63$ & 0.027 \\
\hline Nodule's maximum diameter (mm) & $19.18 \pm 5.45$ & $20.31 \pm 5.54$ & 0.328 \\
\hline Creatinine $(\mu \mathrm{mol} / \mathrm{L})$ & $883.53 \pm 251.86$ & $847.30 \pm 216.04$ & 0.445 \\
\hline Uric acid $(\mu \mathrm{mol} / \mathrm{L})$ & $418.36 \pm 118.53$ & $416.72 \pm 103.97$ & 0.942 \\
\hline Albumin(g/L) & $36.74 \pm 4.64$ & $36.39 \pm 4.18$ & 0.698 \\
\hline Haemoglobin $(\mathrm{g} / \mathrm{L})$ & $107.36 \pm 18.68$ & $101.02 \pm 23.01$ & 0.132 \\
\hline $\mathrm{CRP}(\mathrm{mg} / \mathrm{L})$ & $7.12 \pm 10.48$ & $11.12 \pm 14.90$ & 0.195 \\
\hline $\operatorname{Tnl}(\mu \mathrm{g} / \mathrm{L})$ & $0.04 \pm 0.05$ & $0.04 \pm 0.04$ & 0.870 \\
\hline $\mathrm{BNP}(\mathrm{pg} / \mathrm{ml})$ & $158.85(73.85-422.5)$ & $230.80(73.65-646.75)$ & 0.316 \\
\hline Baseline iPTH $(\mathrm{pg} / \mathrm{mL})$ & $1857.47 \pm 812.34$ & $1747.31 \pm 924.09$ & 0.527 \\
\hline Calcium (mmol/L) & $2.47 \pm 0.18$ & $2.48 \pm 0.24$ & 0.884 \\
\hline Phosphate(mmol/L) & $2.33 \pm 0.53$ & $2.22 \pm 0.59$ & 0.331 \\
\hline $\mathrm{bALP}(\mathrm{U} / \mathrm{L})$ & $132.31 \pm 33.83$ & $146.07 \pm 62.65$ & 0.246 \\
\hline$\beta-C T x(p g / m l)$ & $5405.12 \pm 951.37$ & $5449.51 \pm 843.51$ & 0.818 \\
\hline N-MID(ng/ml) & $289.69 \pm 391.48$ & $242.34 \pm 56.58$ & 0.451 \\
\hline tPINP(ng/ml) & $1104.22 \pm 296.67$ & $1072.29 \pm 260.13$ & 0.595 \\
\hline $25(\mathrm{OH}) \mathrm{D}(\mathrm{ng} / \mathrm{ml})$ & $21.82 \pm 10.49$ & $20.96 \pm 13.40$ & 0.730 \\
\hline Preoperative clinical symptoms, yes & $62.3 \%$ & $53.2 \%$ & 0.359 \\
\hline Osteoporosis, yes & $38.1 \%$ & $42.3 \%$ & 0.706 \\
\hline Carotid atherosclerosis, yes & $92.3 \%$ & $76.2 \%$ & 0.123 \\
\hline
\end{tabular}

Abbreviations: PTX, Parathyroidectomy; RFA: Radiofrequency Ablation; CRP, C-reactive protein; Tnl, Troponin I; BNP, B-type natriuretic peptide; iPTH, intact parathyroid hormone; bALP, bone-specific alkaline 
phosphatase; $\beta$-CTx, Beta C-terminal cross-linked telopeptides of type『collagen; N-MID, N-terminal osteocalcin; tPINP, total type I collagen N-terminal propeptide

\section{Outcomes}

Primary outcomes: At the time of discharge, $12.0 \%$ of patients in the PTX group and $32.6 \%$ of the patients in the RFA group had iPTH concentrations within the target range $(124-558 \mathrm{pg} / \mathrm{mL})(P=0.015)$. The majority of patients in both groups had low iPTH concentrations $(<124 \mathrm{pg} / \mathrm{mL})$. In addition, the iPTH concentrations of $25.8 \%$ of patients in the PTX group and $51.3 \%$ of the RFA group achieved the recommended goal at the study endpoint $(P=0.031) .58 .1 \%$ of patients in the PTX group who didn't achieve target values still had low iPTH concentrations. (Table 2)

Table 2. Proportion of patients achieving and not achieving the target range iPTH concentration by treatment groups.

\begin{tabular}{|llll|}
\hline iPTH level $(\mathrm{pg} / \mathrm{mL})$ & PTX Group & RFA Group & P value \\
\hline Discharge after treatment & & & 0.051 \\
\hline 124-558, No. $(\%)$ & $6(12.0)$ & $15(32.6)$ & 0.015 \\
\hline$<124$, No. $(\%)$ & $40(80.0)$ & $28(60.9)$ & \\
\hline$>558$, No. $(\%)$ & $4(8.0)$ & $3(6.5)$ & \\
\hline Endpoint & & & 0.054 \\
\hline $124-558$, No. $(\%)$ & $8(25.8)$ & $20(51.3)$ & 0.031 \\
\hline$<124$, No. $(\%)$ & $18(58.1)$ & $12(30.8)$ & \\
\hline$>558$, No. $(\%)$ & $5(16.1)$ & $7(17.9)$ & \\
\hline
\end{tabular}

At the end of our study, there were a total of 15 patients lost to follow-up, of which 12 were in the PTX group and 3 were in the RFA group. And 12 patients died in total during the follow-up period. The allcause mortality of the PTX group and the RFA group were 14.6\% (6/41) and 13.6\% (6/44), respectively. According to the Kaplan-Meier survival curve, there was no statistical difference in long-term survival rates in the two groups $(\mathrm{P}=0.902)$ (Figure 1). A total of 10 patients relapsed, and the recurrence rates were $9.8 \%(4 / 41)$ and $13.6 \%(6 / 44)$ in the PTX and RFA group $(P=0.579)$. There was no significant difference in the cumulative response rate between these two groups $(P=0.141)$ (Figure 2).

Secondary outcomes: iPTH concentrations in both groups decreased sharply from baseline after therapy. Mean iPTH concentrations in PTX group and RFA group immediately after treatment were 82.30 \pm 163.21 $\mathrm{pg} / \mathrm{mL}$ and $279.96 \pm 306.57 \mathrm{pg} / \mathrm{mL}$, respectively $(P<0.001)$. iPTH levels reached a small peak in 6 months in both groups, and there was no significant difference in the trend of these over time between the two 
groups from discharge to 24 months $(P>0.05)$ (Figure 3a). The postoperative serum calcium and phosphorus concentrations of the two groups were significantly lower than the baseline. The average calcium concentration of the RFA group at discharge was the lowest value of $2.04 \pm 0.33 \mathrm{mmol} / \mathrm{L}$, while it dropped to the lowest of $2.01 \pm 0.38 \mathrm{mmol} / \mathrm{L}$ at 1 month in the PTX group. The lowest phosphorus concentrations of the PTX and RFA groups were in $1 \mathrm{month}$, and were $1.00 \pm 0.23 \mathrm{mmol} / \mathrm{L}$ and $1.16 \pm 0.40 \mathrm{mmol} / \mathrm{L}$ in that order. However, the comparison of the mean calcium and phosphorus concentrations at different follow-up times was no statistical difference between the two groups $(P>0.05)$ (Figure 3b, $\underline{3 c}$ ).

\section{Adverse events and complications}

Hoarseness occurred in 4 and 6 cases respectively in the PTX and RFA groups( $P=0.385)$. And hematoma occurred in 3 and 1 cases in the PTX and RFA groups $(P=0.368)$. The incidence of hypocalcemia in the RFA group was $55.3 \%$, but there was no significant difference compared with the PTX group, which was 43.1\% $(\mathrm{P}=0.228)$. SH occurred in 10 patients, including 4 in the PTX group, and 6 in the RFA group $(P=0.421)$. The incidence fever or infection in the PTX group was obviously more than of the others $(P<0.001)$. There was a significant difference in postoperative CRP levels between the two groups $(P=0.015)$. (Table 3$)$

Table 3. Comparison of safe between PTX and RFA group

\begin{tabular}{|llll|}
\hline Parameters & PTX Group( $\mathrm{n}=53)$ & RFA group $(\mathrm{n}=47)$ & P value \\
\hline Total hospital stay(days) & $15.49 \pm 8.57$ & $11.60 \pm 4.52$ & 0.006 \\
\hline Postoperative hospital stay(days) & $7.85 \pm 5.92$ & $4.53 \pm 2.98$ & 0.001 \\
\hline CRP after treatment & $34.20(19.00-88.55)$ & $24.00(6.50-38.40)$ & 0.015 \\
\hline Complications & & & \\
\hline Hoarseness, No.(\%) & $4(7.5)$ & $6(12.8)$ & 0.385 \\
\hline Hematoma, No.(\%) & $3(5.7)$ & $1(2.1)$ & 0.368 \\
\hline Fever/infection, No.(\%) & $21(39.6)$ & $3(6.4)$ & $<0.001$ \\
\hline Hypocalcemia, No.(\%) & $22(43.1)$ & $26(55.3)$ & 0.228 \\
\hline Severe hypocalcemia, No.(\%) & $4(7.8)$ & $6(12.8)$ & 0.421 \\
\hline
\end{tabular}

Hypocalcemia<2.0mmol/L; Severe hypocalcemia< $1.8 \mathrm{mmol} / \mathrm{L}$

Compared with the PTX group, the total hospital stays in the RFA group ( $15.49 \pm 8.57$ days vs $11.60 \pm 4.52$ days, $\mathrm{P}=0.006)$ and postoperative hospital stays $(7.85 \pm 5.92$ days vs $4.53 \pm 2.98$ days, $\mathrm{P}=0.001)$ were significantly less. Furthermore, the RFA group was analyzed by subgroups. There was no statistical difference between the single-session RFA group and the two-session RFA group in terms of total and 
postoperative hospitalization time ( $10.73 \pm 4.17$ days vs $12.67 \pm 4.14$ days, $\mathrm{P}=0.147 ; 4.62 \pm 3.70$ Days vs $4.43 \pm 1.83$ days, $P=0.834)$. However, in terms of operating expenses, the single-session ablation of $1447.70 \pm 41.88 \$$ was less than parathyroidectomy of $1633.85 \pm 258.84 \$$, but the cost of two-session ablation was $2820.25 \pm 1.99 \$$.

\section{Risk factors of hypocalcemia}

Univariate analysis showed that lower age $(\mathrm{P}=0.030)$ and baseline serum calcium concentrations $(P=0.009)$, higher baseline iPTH levels $(P=0.019)$, greater reductions of iPTH levels in $D 1(P=0.006)$, higher bALP levels $(P<0.001)$ were associated with a higher risk of hypocalcemia (Table 4$)$. When the cut-off point of bALP level was set as $115 \mathrm{U} / \mathrm{L}$, the area under the ROC curve was 0.762 , the sensitivity was $80.0 \%$, and the specificity was $63.6 \%$ (Figure 4). The results of binary logistic regression analysis showed that OR=1.033 (1.013-1.052), for every increase of bALP levels(1U/L), the risk of hypocalcemia increased by $3.3 \%$.

Table 4. ROC curves in patients with hypocalcemia

\begin{tabular}{|llllll|}
\hline Variables & Cut-off & AUC & Sensitivity & Specificity & P value \\
\hline Baseline iPTH $(\mathrm{pg} / \mathrm{mL})$ & 1210 & 0.638 & 0.833 & 0.36 & 0.019 \\
\hline Reduction of $\mathrm{iPTH}(\mathrm{pg} / \mathrm{mL})$ & 894.9 & 0.667 & 0.979 & 0.304 & 0.006 \\
\hline Serum calcium $(\mathrm{mmol} / \mathrm{L})$ & 2.61 & 0.654 & 0.420 & 0.896 & 0.009 \\
\hline BALP(U/L)* & 115 & 0.762 & 0.800 & 0.636 & $<0.001$ \\
\hline Age(years) & 47.5 & 0.627 & 0.700 & 0.542 & 0.030 \\
\hline
\end{tabular}

$\mathrm{AUC}^{*}>0.7$

\section{Discussion}

In our retrospective study, a total of 100 maintenance dialysis patients were treated with SHPT. The baseline characteristics of the two groups were almost the same. Since our hospital had performed PTX as early as 2014, and US-guided RFA began in 2017, the follow-up times were slightly different. In addition, more hyperplastic parathyroid nodules were founded in the PTX group because of the higher visibility.

Resulting in decreased kidney function, disordered calcium-phosphorus-vitamin D metabolism, and increased synthesis and secretion of PTH, it leads to abnormal bone turnover, mineralization, vascular or soft tissue calcification, which is clinically diagnosed as CKD-MBD ${ }^{1,22}$. This study showed about $60 \%$ of participants had clinical symptoms such as ostealgia or arthralgia, cutaneous pruritus, skeleton distortion, and calcinosis cutis, etc. Bone-derived turnover markers such as N-MID, $\beta$-CTx, tP1NP and bALP were significantly higher than normal. Examinations suggested some patients had osteoporosis and 
carotid arteriosclerosis. Therefore, KDIGO guidelines ${ }^{21}$ recommended the treatment of CKD-MBD targeted and maintained at proper ranges of $\mathrm{IPTH}$, calcium and phosphorus concentrations, and suggested nonpharmacological therapy for patients with severe hyperparathyroidism.

Most patients in the PTX group accepted total PTX with AT in our hospital. The advantage of this operation is that the function of the transplanted glands can be monitored by measuring the iPTH levels of the bilateral forearms ${ }^{23}$. Our study showed that PTX was effective in the treatment of SHPT, which was consistent with the results of previous studies ${ }^{4,24,25}$. Patients who cannot tolerate surgery can be treated with ultrasound-guided thermal ablation. Several studies compared the efficacy of MWA[7,10,26-29] and PTX for SHPT. There is no clear conclusion which method is better. A meta-analysis ${ }^{29}$ revealed that both thermal ablation and PTX were effective treatment options for SHPT, and thermal ablation increased the risk of recurrence. But there was no significant difference in the cumulative response rate between the two groups in our study. And there are no definite criteria for choosing which ablation method to treat SHPT at present. Some studies ${ }^{6,30}$ compared the effects of RFA and MWA in the treatment of primary hyperparathyroidism and proved that both were safe and effective. Some studies ${ }^{31-33}$ had applied ethanol ablation (EA) to parathyroid adenoma, which can decrease iPTH levels and adenoma volumes, but it is prone to recurrence and requires multiple injections. There is no consensus about the amount of ethanol used and the ideal treatment interval. It was ${ }^{34,35}$ reported that RFA appeared to be superior to other ablation types in reducing the volume of thyroid nodules and EA was more effective in the treatment of cyst lesions. There are few studies reported RFA had been applied for SHPT. The results of this study were consistent with previous retrospective cohort study ${ }^{12}$ of 56 patients who underwent USguided RFA, showed that iPTH concentrations decreased significantly.

This study compared the clinical efficacy of RFA and PTX, and revealed that the iPTH concentrations were rapid decreased, and there were no differences in the iPTH, calcium and phosphorus fluctuation curves between the two groups during the follow-up period. At the study endpoint, the proportion of the patients within the target range of iPTH in the RFA group was significantly higher ( $51.3 \%$ vs $25.8 \%$, $\mathrm{P}=0.031$ ). And more than half of the patients underwent PTX remained low iPTH levels. Continuously low PTH levels will lead to permanent hypoparathyroidism, which is associated with adynamic bone disease $^{36}$, reduced bone formation, and obvious bone pain or fractures. Thus, it is very important to maintain ideal iPTH levels in order to be in the normal bone turnover state ${ }^{37}$. As we all known, PTX contributes to reduce the risks of all-cause mortality in CKD patients with SHPT ${ }^{4,24}$, but there are few studies about whether RFA is benefit to long-term prognosis. The results of the survival analysis in the present study showed that there was no difference in all-cause mortality between two groups $(\mathrm{P}=0.902)$. Other researchers ${ }^{14}$ reported that there was no significant difference in the cumulative death rate between the MWA and PTX groups.

Hypocalcemia is a common complication after the invasive treatment of SHPT ${ }^{5,18}$, due to the abrupt decline of iPTH. Our results showed that although there was no significant difference about the postoperative hypocalcemia between the two groups, the serum calcium levels of the PTX group 
continued to decrease until to 1 month after surgery, which was associated with the relatively low iPTH concentrations. bALP ${ }^{38}$ is an extracellular enzyme of osteoblasts, which is a biomarker of osteoblast maturity and bone turnover, and it is more accurate and specific than ALP. We found that the postoperative low calcium level of patients was significantly correlated with the higher baseline bALP level. Previous analysis indicated that preoperative ALP level as a risk factor for hypocalcemia $5,18,19$. In our study, patients with baseline bALP concentration more than $115 \mathrm{U} / \mathrm{L}$ were prone to hypocalcemia, the sensitivity was $80.0 \%$, and the specificity was $63.6 \%$.

The incidences of the other complications including hoarseness and hematoma were similar between two groups, but the incidence of infection in the PTX group was significantly higher $(P<0.001)$. PTX requires general anesthesia and longer operation time, and it is traumatic and invasive ${ }^{23}$. The hospital stay is relatively longer due to the prolonged recovery time. In terms of procedure cost, single-session RFA had a weak superiority than PTX. The advantages of US-guided RFA are minimally invasive, fast, and reproducible, and it is no less than PTX from the perspective of safety and efficacy.

There were still some limitations in this study. First, the number of patients enrolled is relatively small, and we need larger samples to explore the efficacy and safety. Second, as a retrospective study, the results may be biased due to some people were lost to follow-up. Prospective studies are needed to verify the therapeutic value of RFA for SHPT and to compare the effects of these two procedures on the prognosis by dynamic assessment. Third, because US-guided RFA was performed by the same sonographer, it may affect the reproducibility.

\section{Conclusion}

In summary, ultrasound-guided RFA is a promising, safe and effective minimally invasive method for severe secondary hyperparathyroidism in maintenance dialysis patients. It may be used as an alternative technique to PTX, and further prospective studies and randomized controlled trials are necessary to confirm it.

\section{Declarations}

\section{Acknowledgement}

I would like to thank all the professors and colleagues who gave me guidance and assistance in the course of the study. Thank my parents and relatives for their support and understanding. In the end, I want to give special thanks to Mr. Yibo Wang and Mr. Zhan Xiao. Their excellent personalities are worthy of my lifelong learning.

\section{Statement of Ethics}

This study protocol was reviewed and approved by the ethical and scientific review board of Zhejiang Provincial People's Hospital, approval number [2021QT330]. And the study has been granted an 
exemption from requiring written informed consent.

\section{Competing interests}

The authors declare no competing interests.

\section{Funding Sources}

There is no funding source for this study.

\section{Author Contribution Statement}

Research idea and study design: M.R., D.N.Z., J.W., Y.M.L., B.L.; data acquisition: M.R., D.N.Z.; data analysis/interpretation: M.R., D.N.Z., J.W., Y.M.L.; statistical analysis: M.R.; manuscript drafting and revision: M.R., D.N.Z.; approval of final version of submitted manuscript: M.R., D.N.Z., J.W., Y.M.L., C.Z.P., W.S., B.L.; agrees to ensure any questions related to the work are appropriately resolved: M.R., D.N.Z., J.W., Y.M.L., C.Z.P., W.S., B.L..

\section{Data Availability Statement}

All data generated or analyzed during this study are included in this article. Further enquiries can be directed to the corresponding author.

\section{References}

1. Cunningham, J., Locatelli, F. \& Rodriguez, M. Secondary hyperparathyroidism: Pathogenesis, disease progression, and therapeutic options. Clinical Journal of the American Society of Nephrology vol. 6 913-921 (2011).

2. Davies, E. W. et al. Health state utilities associated with major clinical events in the context of secondary hyperparathyroidism and chronic kidney disease requiring dialysis. Health and Quality of Life Outcomes 13, (2015).

3. Tentori, F. et al. Recent changes in therapeutic approaches and association with outcomes among patients with secondary hyperparathyroidism on chronic hemodialysis: The DOPPS study. Clinical Journal of the American Society of Nephrology 10, 98-109 (2015).

4. Ivarsson, K. M. et al. The effect of parathyroidectomy on patient survival in secondary hyperparathyroidism. Nephrology Dialysis Transplantation 30, 2027-2033 (2015).

5. Liu, J., Huang, Q., Yang, M., Huang, L. \& Zhang, L. Risk factors predicting severe hypocalcemia after total parathyroidectomy without autotransplantation in patients with secondary hyperparathyroidism. Journal of International Medical Research 48, (2020).

6. Wei, Y. et al. Microwave ablation versus radiofrequency ablation for primary hyperparathyroidism: a multicenter retrospective study. International Journal of Hyperthermia 38, 1023-1030 (2021). 
7. Hu, Z. et al. Feasibility and safety of ultrasound-guided percutaneous microwave ablation for tertiary hyperparathyroidism. International Journal of Hyperthermia 36, 1129-1136 (2019).

8. Wang, R., Jiang, T., Chen, Z. \& Chen, J. Regression of calcinosis following treatment with radiofrequency thermoablation for severe secondary hyperparathyroidism in a hemodialysis patient. Internal Medicine 52, 583-587 (2013).

9. Kim, H.-S. et al. Delayed Surgery for Parathyroid Adenoma Misdiagnosed as a Thyroid Nodule and Treated with Radiofrequency Ablation. Endocrinology and Metabolism 28, 231 (2013).

10. Wang, G. et al. Microwave ablation: an effective treatment for mild-to-moderate secondary hyperparathyroidism in patients undergoing haemodialysis. International Journal of Hyperthermia 33, 946-952 (2017).

11. Cao, X. J. et al. Efficacy and safety of microwave ablation treatment for secondary hyperparathyroidism: systematic review and meta-analysis. International Journal of Hyperthermia vol. 37 316-323 (2020).

12. Peng, C. et al. Efficacy and safety of ultrasound-guided radiofrequency ablation of hyperplastic parathyroid gland for secondary hyperparathyroidism associated with chronic kidney disease. Head and Neck 39, 564-571 (2017).

13. Liu, F. et al. Comparison of ultrasound-guided percutaneous microwave ablation and parathyroidectomy for primary hyperparathyroidism. International Journal of Hyperthermia 36, 835840 (2019).

14. Diao, Z. et al. Microwave ablation versus parathyroidectomy for severe secondary hyperparathyroidism in patients on hemodialysis: a retrospective multicenter study. International Journal of Hyperthermia 38, 213-219 (2021).

15. Jiang, B. et al. Microwave ablation vs. parathyroidectomy for secondary hyperparathyroidism in maintenance hemodialysis patients. Hemodialysis International 23, 247-253 (2019).

16. Kritmetapak, K. et al. Incidence of and risk factors for post-parathyroidectomy hungry bone syndrome in patients with secondary hyperparathyroidism. Renal Failure 42, 1118-1126 (2020).

17. Wei, Y. et al. Hypocalcemia after ultrasound-guided microwave ablation and total parathyroidectomy for secondary hyperparathyroidism: a retrospective study. International Journal of Hyperthermia 37, 819-825 (2020).

18. Zeng, Z. et al. Efficacy of Ultrasound-guided Radiofrequency Ablation of Parathyroid Hyperplasia: Single Session vs. Two-Session for Effect on Hypocalcemia. Scientific Reports 10, (2020).

19. Wei, Y., Peng, L. li, Zhao, Z. long, Li, Y. \& Yu, M. an. Risk Factors of Severe Hypocalcemia After USGuided Percutaneous Microwave Ablation of the Parathyroid Gland in Patients with Secondary Hyperparathyroidism. Journal of Bone and Mineral Research 35, 691-697 (2020).

20. Viaene, L. et al. Calcium requirements after parathyroidectomy in patients with refractory secondary hyperparathyroidism. Nephron - Clinical Practice 110, (2008).

21. Indd, K. KDIGO 2017 Clinical Practice Guideline Update for the Diagnosis, Evaluation, Prevention, and Treatment of Chronic Kidney Disease-Mineral and Bone Disorder (CKD-MBD). (2017). 
22. Fukagawa, M. et al. Clinical practice guideline for the management of chronic kidney disease-mineral and bone disorder. Therapeutic Apheresis and Dialysis 17, 247-288 (2013).

23. van der Plas, W. Y. et al. Safety and efficacy of subtotal or total parathyroidectomy for patients with secondary or tertiary hyperparathyroidism in four academic centers in the Netherlands. Langenbeck's Archives of Surgery 403, 999-1005 (2018).

24. Chen, L. et al. Long-term mortality after parathyroidectomy among chronic kidney disease patients with secondary hyperparathyroidism: a systematic review and meta-analysis. Renal Failure 38, 1050-1058 (2016).

25. Wan, J., Li, W. \& Zhong, Y. Parathyroidectomy decreases serum intact parathyroid hormone and calcium levels and prolongs overall survival in elderly hemodialysis patients with severe secondary hyperparathyroidism. Journal of Clinical Laboratory Analysis 33, (2019).

26. Li, X., An, C., Yu, M. \& Peng, L. Us-guided microwave ablation for secondary hyperparathyroidism in patients after renal transplantation: A pilot study. International Journal of Hyperthermia 36, 322-327 (2019).

27. Zhuo, L. et al. Microwave ablation of hyperplastic parathyroid glands is a treatment option for endstage renal disease patients ineligible for surgical resection. International Journal of Hyperthermia 36, 29-35 (2019).

28. Zhuo, L. et al. Us-guided microwave ablation of hyperplastic parathyroid glands: Safety and effcacy in patients with end-stage renal disease - A pilot study. Radiology 282, 576-584 (2017).

29. Gong, L., Tang, W., Lu, J. \& Xu, W. Thermal ablation versus parathyroidectomy for secondary hyperparathyroidism: A meta-analysis. International Journal of Surgery vol. 70 13-18 (2019).

30. Wei, Y. et al. Effectiveness and Safety of Thermal Ablation in the Treatment of Primary Hyperparathyroidism: A Multicenter Study. The Journal of Clinical Endocrinology \& Metabolism 106, 2707-2717 (2021).

31. Fletcher1, S. et al. Nephrology Dialysis Transplantation Assessment of ultrasound guided percutaneous ethanol injection and parathyroidectomy in patients with tertiary hyperparathyroidism. Nephrol Dial Transplant vol. 13 (1998).

32. Yazdani, A. et al. Ultrasound-guided ethanol injection for the treatment of parathyroid adenoma: A prospective self-controlled study. Journal of Research in Medical Sciences 25, 93 (2020).

33. Schamp, S. et al. Ultrasound-guided Percutaneous Ethanol Ablation of Parathyroid Hyperplasia: Preliminary Experience in Patients on Chronic Dialysis. Ultraschall in der Medizin 25, 131-136 (2004).

34. He, L. et al. Comparative efficacy of different ultrasound-guided ablation for the treatment of benign thyroid nodules: Systematic review and network meta-analysis of randomized controlled trials. PLoS ONE 16, (2021).

35. Ha, E. J., Baek, J. H. \& Baek, S. M. Minimally invasive treatment for benign parathyroid lesions: Treatment efficacy and safety based on nodule characteristics. Korean Journal of Radiology 21, 1388-1397 (2020). 
36. Hsu, C. Y., Chen, L. R. \& Chen, K. H. Osteoporosis in patients with chronic kidney diseases: A systemic review. International Journal of Molecular Sciences vol. 21 1-24 (2020).

37. Ott, S. M., Malluche, H. H., Jorgetti, V. \& Elder, G. J. Importance of bone turnover for therapeutic decisions in patients with CKD-MBD. Kidney Internationa/ vol. 100 502-505 (2021).

38. Ginsberg, C. \& IX, J. H. Diagnosis and Management of Osteoporosis in Advanced Kidney Disease: A Review. American Journal of Kidney Diseases (2021) doi:10.1053/j.ajkd.2021.06.031.

\section{Figures}

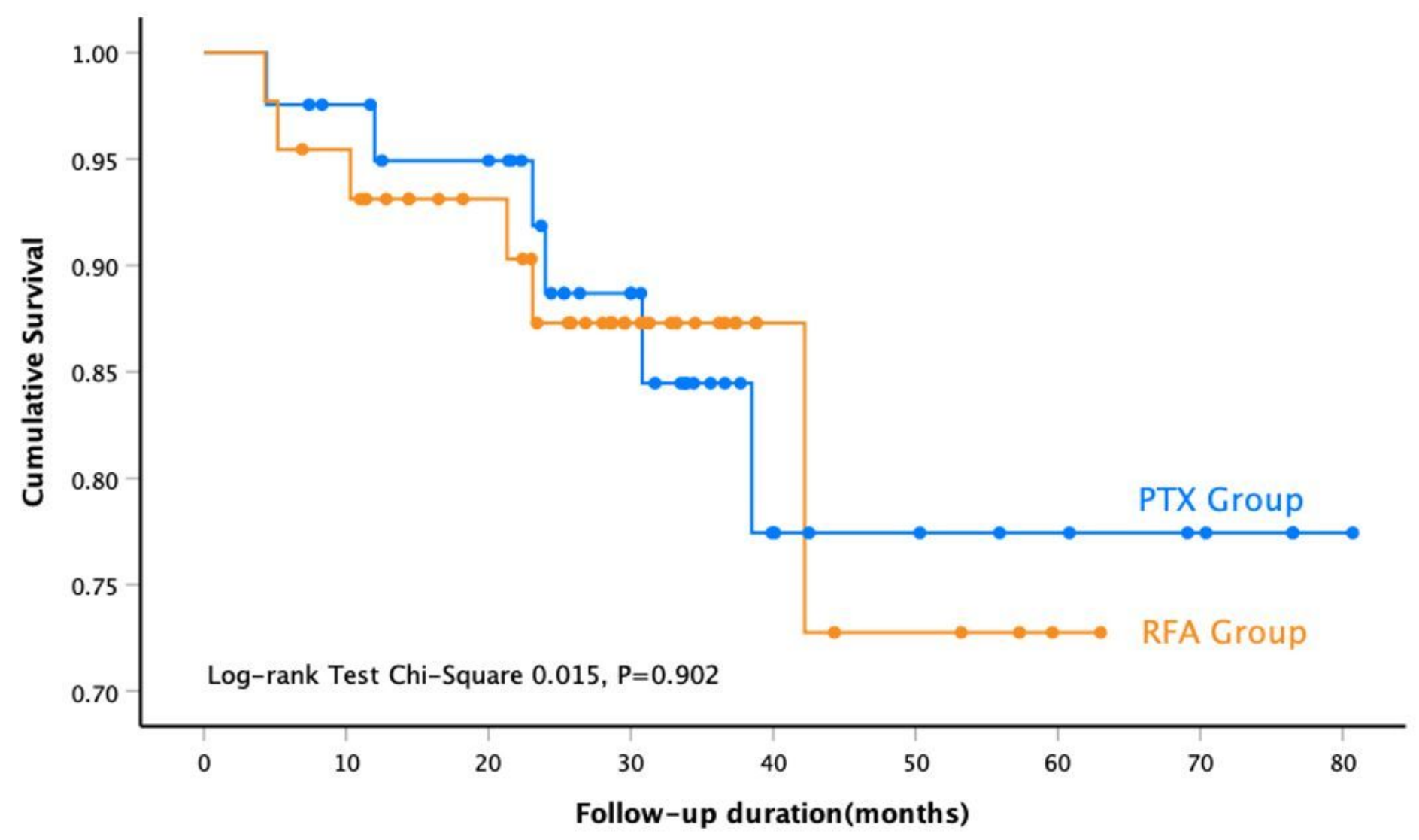

Figure 1

Survival analysis of all-cause mortality after parathyroidectomy or ultrasound-guided radiofrequency ablation. 


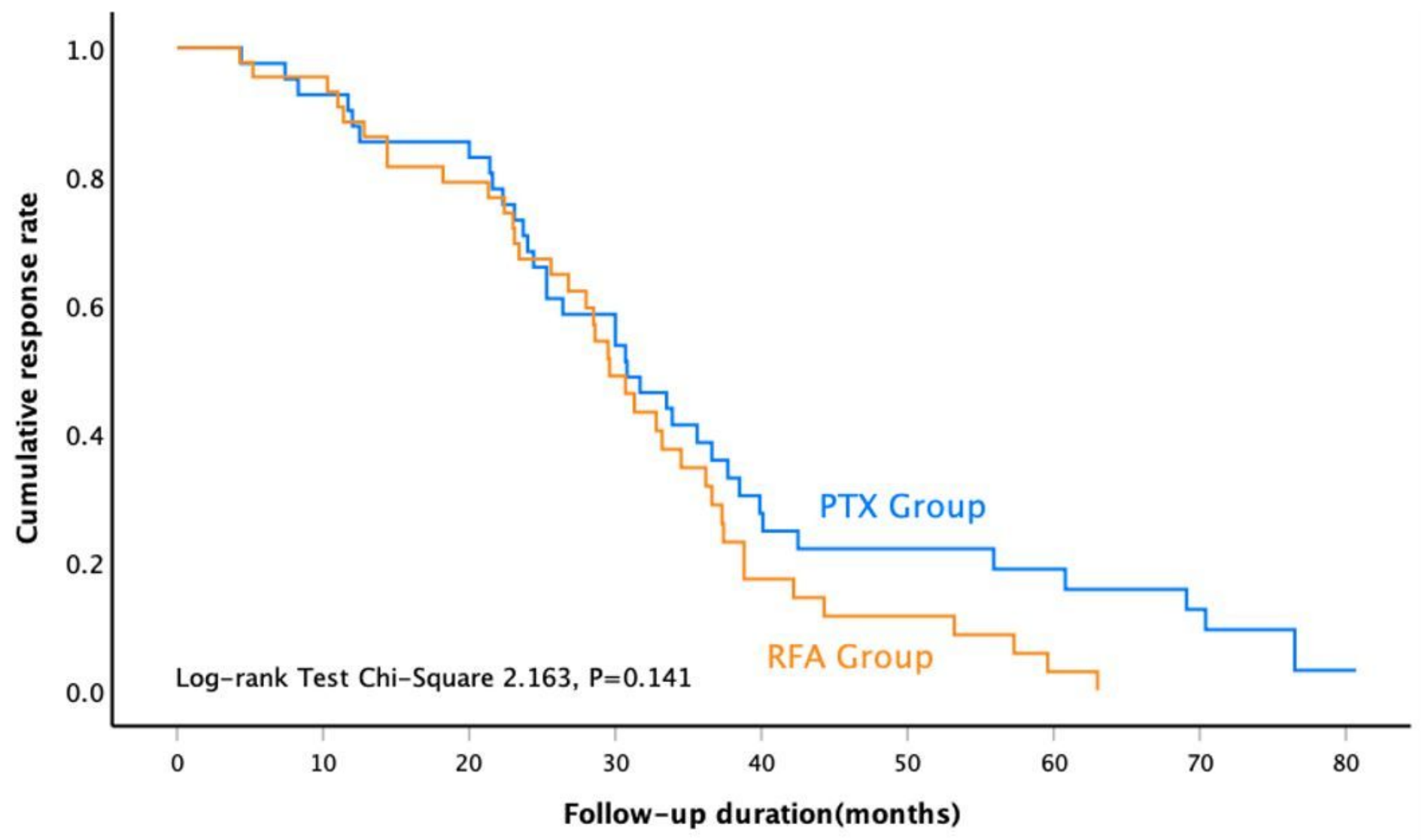

Figure 2

Cumulative response rate of the PTX and RFA group
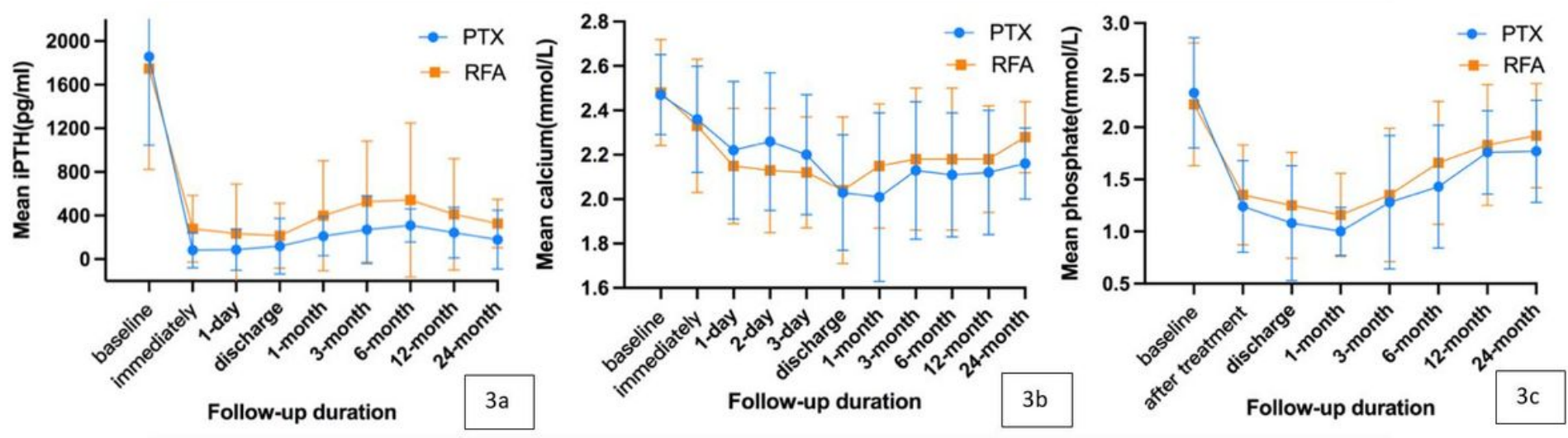

Figure 3

Mean iPTH concentrations (a), calcium (b), and phosphate (c) concentrations in patients treated with parathyroidectomy or ultrasound-guided radiofrequency ablation during the study period. iPTH: intact parathyroid hormone. 


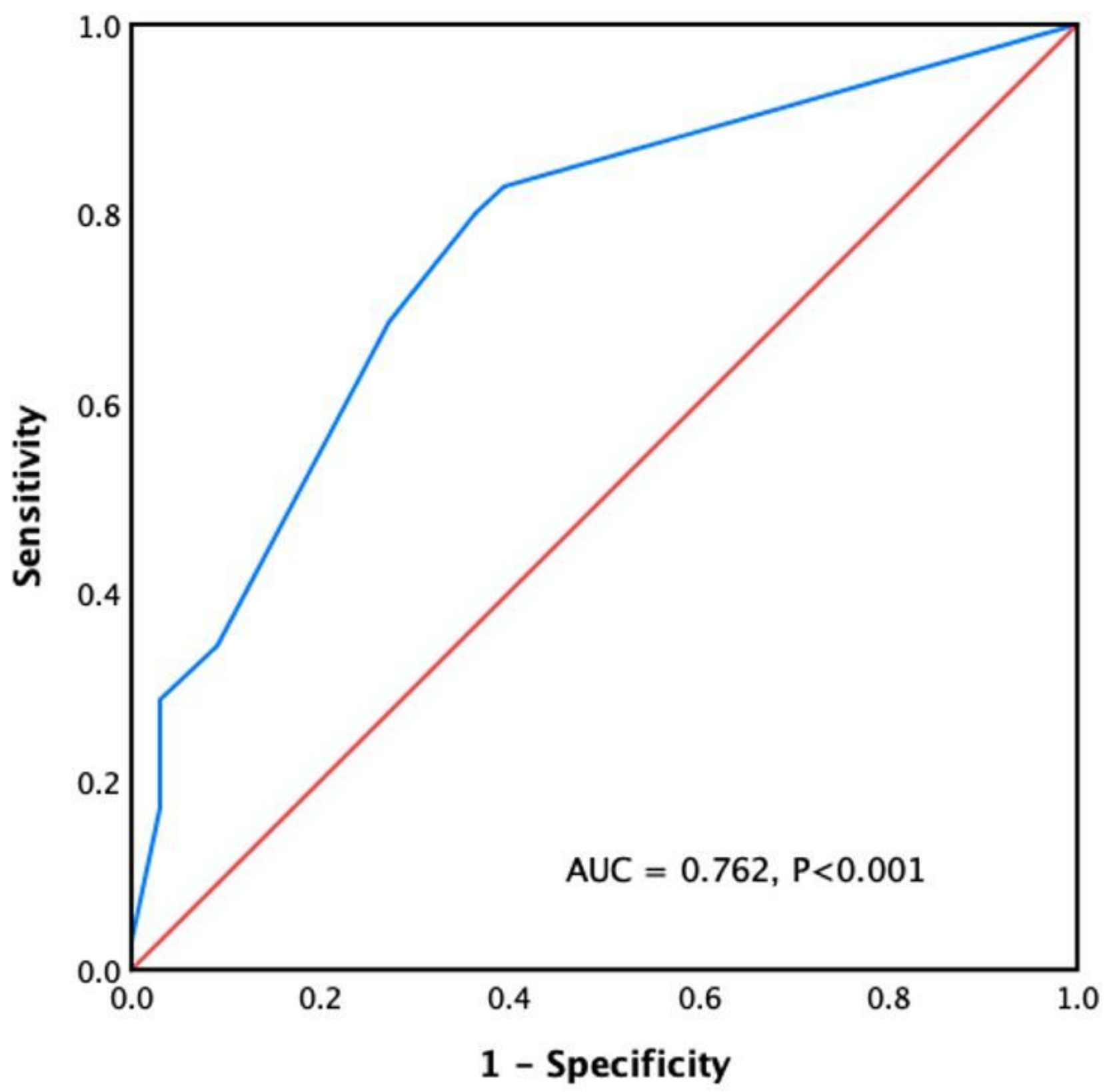

Figure 4

ROC curve of bone-specific alkaline phosphatase (bALP) shows area under the curve of 0.762 , sensitivity of $80 \%$ and specificity of $63.6 \%$ using bALP cutoff point as $115 \mathrm{mmol} / \mathrm{L}$. 